Revue d'histoire de l'enfance « irrégulière »

Le Temps de l'histoire

$3 \mid 2000$

L'enfant de justice pendant la guerre et l'immédiat après-guerre

\title{
L'Université et l'enfance délinquante : 1939-1945
}

Jean-Jacques Yvorel

\section{OpenEdition}

Journals

Édition électronique

URL : http://journals.openedition.org/rhei/74

DOI : $10.4000 /$ rhei. 74

ISBN : 978-2-7535-1640-3

ISSN : $1777-540 \mathrm{X}$

Éditeur

Presses universitaires de Rennes

Édition imprimée

Date de publication : 15 novembre 2000

Pagination : 137-157

ISSN : $1287-2431$

Référence électronique

Jean-Jacques Yvorel, « L'Université et l'enfance délinquante : 1939-1945 », Revue d'histoire de l'enfance « irrégulière » [En ligne], 3 | 2000, mis en ligne le 30 avril 2007, consulté le 03 décembre 2020. URL http://journals.openedition.org/rhei/74 ; DOI : https://doi.org/10.4000/rhei.74 


\section{L'Université et l'enfance délinquante : 1939-1945}

\section{Introduction}

Entre 1939 et 1945, une petite quarantaine de thèses se rapportant à l'enfance délinquante sont soutenues dans les universités françaises qui, malgré les événements, continuent de fonctionner. Ce sont ces discours académiques que nous avons cherché à analyser. Constitués sur un même registre, ils forment un corpus relativement homogène et s'inscrivent dans une tradition qui permet des comparaisons.

Nous avons repéré trente-sept thèses consacrées à l'enfance délinquante publiées entre 1939 et 1945 [voir liste en fin d'article]. Notre mode de recherche empirique ne permet pas de prétendre à une quelconque exhaustivité. Nous avons utilisé, pour établir notre liste, le Catalogue des thèses et écrits académiques, qui ne contient que les noms des auteurs et les titres des doctorats soutenus dans les facultés françaises. Outre les oublis toujours possibles lors de la lecture de sept volumes de références, l'absence d'index thématique ne permet pas de détecter les travaux consacrés à l'enfant de justice quand le titre n'est pas à cet égard explicite. Aussi avons-nous complété nos investigations en utilisant la bibliographie établie par JeanClaude Farcy ${ }^{(2)}$ et celle due à Monique Brisset et Éric Pierre, qui complète la réédition de l'ouvrage d'Henri Gaillac sur Les maisons de correction. ${ }^{(3)}$ D'autre part, nous n'avons pas eu accès à tous ces textes ; l'état de conservation de certains documents ne permet pas leur communication et ils n'ont pas encore été transférés sur microfiches. De plus, toutes les thèses de province soutenues entre 1939 et 1945 n'ont pas été déposées à la Bibliothèque nationale. Nous avons finalement consulté 21 textes.

Notre étude s'ouvre sur une présentation des caractéristiques d'ensemble de ces écrits. Ce traitement global nous a permis de distinguer

\section{Jean-Jacques Yvorel $^{(1)}$}

(1) Historien, chargé d'études au CNFE-PJJ, Vaucresson.

(2) Jean-Claude FARCY, Deux siècles d'histoire de la Justice en France, 1789-1989, Notices bibliographiques, Paris,

CNRS éditions, 1996, CD-ROM.

(3) Henri GAILLAC, Les maisons de correction, 1830-1945, Paris, Cujas, 1991, 463 p. La bibliographie occupe les pages 379 à 463.

Jean-Jacques Yvorel / pp. 137 à 157 
(4) Par exemple Louis

BEGON, Étude du

meurtre chez l'adolescent, Paris, E. Le François, 1940, $32 \mathrm{p}$.

(5) C'est le cas de la thèse d'Émile CHAUVEL, Contribution à l'étude médicale et sociale de la délinquance juvénile, Paris, 18 p. dactylographiées.

(6) Philippe REY-

HERME, Les colonies de vacances en France, Paris, Fleurus, 1961, 3 vol.

(7) Nous savons que, jusqu’à la seconde guerre mondiale, les rares femmes médecins furent majoritairement confinées dans la pédiatrie. Bénédicte VERGEZ, Le monde des médecins au XXème siècle, Bruxelles, Complexe, 1996, pp. 63-64. trois niveaux de discours dont nous analyserons les développements dans une seconde partie. Nous verrons enfin si les circonstances marquent les propos des impétrants docteurs ou s'ils s'inscrivent dans une continuité avec les interprétations précédentes de la délinquance juvénile, celles de l'Entre-deux-guerres, voire du XIXème siècle.

\section{I) CARACTÈRES GÉNÉRAUX DES DISCOURS ACADÉMIQUES}

Très courtes au début du XIXème siècle, les thèses tendent à "grossir". Les juristes et les "littéraires" sont généralement plus prolixes que les médecins, puisque les premiers rédigent entre 150 et 340 pages (moyenne 210 p.) contre 30 à 130 pages pour les seconds (moyenne 90 p.). Notons cependant que, dans notre sélection, certains textes sont très courts $^{(4)}$ et il arrive même que, contrairement à l'usage, ils ne soient pas imprimés ${ }^{(5)}$; il faut voir là une conséquence des difficultés de l'époque et un effet des pénuries.

A l'exception de ceux qui ont suivi une carrière publique comme Fernand Joubrel, un des animateurs de l'éducation spécialisée, ou Philippe Rey-Herme, futur professeur de l'Institut catholique et auteur d'une histoire des colonies de vacances, ${ }^{(6)}$ nous ignorons presque tout des auteurs. En nous fiant aux prénoms, nous avons simplement pu constater la présence de neuf femmes parmi les trente-sept rédacteurs. Au regard du nombre de doctorantes qui, avant guerre, ne dépasse pas $15 \%$, il y a donc une réelle sur-représentation. L'enfance et le "social" sont bien des domaines féminins. ${ }^{(7)}$

La thèse peut être appréhendée comme un "genre littéraire", mais un genre dont les "règles narratives" seraient très liées au fonctionnement des facultés. En principe, il ne convient pas dans cet exercice universitaire, pour la période considérée, de faire preuve d'originalité ou de chercher à révolutionner le savoir scientifique : le doctorat est d'abord un acte d'allégeance à des maîtres qui sont parfois imités jusqu'à la caricature. C'est rarement dans ce type de littérature que nous trouvons, 
pour le XIXème siècle et la première moitié du XXème siècle, les ruptures épistémologiques et autres révolutions de la pensée. Par contre, ces mètres linéaires d'imprimés, saisis dans leur totalité, sans s'arrêter aux quelques textes marquants qui peuvent émerger par exception, constituent un bon reflet des idées et des théories professées dans les amphithéâtres par les universitaires les mieux installés.

\section{I-1 L'apparente domination du discours médical}

Durant la période que nous étudions, il n'existe que des thèses de droit, de médecine, de pharmacie, de lettres et de sciences. Les travaux d'histoire, de philosophie, de sciences sociales ou de psychologie sont rattachés au doctorat ès lettres. Les économistes voient leur sort lié à celui des juristes. Le doctorat ès sciences englobe la biologie et les sciences naturelles, ainsi que tout ce que nous nommons aujourd'hui "sciences dures". En recherchant dans le Catalogue des thèses et écrits académiques les mémoires consacrés à "l'enfant de justice", nous pensions que les facultés de droit fourniraient le plus grand nombre de références. Or l'essentiel de la littérature doctorale a été l'œuvre des disciples d'Esculape et non des héritiers de Thémis. Les lettres, la philosophie pour être plus précis, fournissent quant à elles deux titres, ${ }^{(5)}$ les sciences et la pharmacie aucun.
(8) Il s'agit des thèses de Philippe REY-

HERME, Quelques aspects du progrès pédagogique dans la réducation de la jeunesse délinquante, Paris, Vrin, 1945, 190 p., et de Mario CARRASCOBARROIS, Théories sur les causes de la criminalité infantile et juvénile, étude critique, Paris, Jouve, 1942, 158 p. Notons que CARRASCO-BARROIS, s'il soutient une thèse de lettres, n'en est pas moins juriste, puisqu'il est avocat, inscrit au barreau de Santiago du Chili.

Répartition chronologique et disciplinaire des thèses consacrées à l'enfant de justice entre 1939 et 1945

\begin{tabular}{c|c|c|c|c|c|c|c|c} 
& 1939 & 1940 & 1941 & 1942 & 1943 & 1944 & 1945 & TOTAL \\
\hline Droit & 0 & 0 & 2 & 3 & 2 & 2 & 3 & 12 \\
\hline Médecine & 4 & 3 & 1 & 1 & 4 & 3 & 7 & 23 \\
\hline Lettres & 0 & 0 & 0 & 1 & 0 & 0 & 1 & 2 \\
\hline Total & 4 & 3 & 3 & 5 & 6 & 5 & 11 & 37
\end{tabular}

L'importance du discours médical dans le champ de la déviance juvénile ne se traduit pas seulement dans cette supériorité numérique des thèses de médecine, mais aussi dans l'usage, par les juristes eux-mêmes, 
(9) Sur la naissance de la pédopsychiatrie, voir Nadine LEFAUCHEUR, "Psychiatrie infantile et délinquance juvénile. Georges Heuyer et la question de la genèse "familiale" de la délinquance", in Laurent MUCCHIELLI, Histoire de la criminologie française, Paris, L'Harmattan, 1994, pp. 313-332.

(10) André PER-

REAU, Le mineur pervers de constitution, contribution à l'étude de l'enfance coupable, Lyon, Bosc Frères \& L. Riou, 1942, $152 \mathrm{p}$.

(11) Ernest DUPRÉ,

"Les perversions instinctives”, Rapport au Congrés des aliénistes de France, Tunis, 1912, rééd. in Pathologie de l'imagination et de l'émotivité, Paris, Logre, 1925 , pp. 357-427.

(12) Georges HEUYER, Enfants anormaux et délinquants juvéniles, Paris, Steinheil, d'un vocabulaire médical emprunté essentiellement à la neuropsychiatrie infantile. Georges Heuyer, le père fondateur de la discipline, est cité par tous les auteurs. ${ }^{(9)}$ Un avocat stagiaire lyonnais consacre même son mémoire au "pervers de constitution" ${ }^{(10)}$ c'est-à-dire à une catégorie clinique créée au début du siècle par l'aliéniste Ernest Dupré ${ }^{(1)}$ et intégrée dans la nosographie pédopsychiatrique d'Heuyer. ${ }^{(12)}$

Cette domination est cependant toute relative car, si les juristes s'aventurent fréquemment sur les terres d'Hippocrate, les hommes de l'art n'hésitent pas à commenter les textes de loi. Georges-Dominique Pesle nous propose même un tour d'horizon des législations étrangères d'une dizaine de pages. Si l'on y ajoute sa présentation de la législation française, c'est presque un quart de cette thèse de médecine qui est consacrée au droit. ${ }^{(13)}$ Mais, surtout, les propositions qui concluent parfois les écrits des thérapeutes sont exclusivement législatives ou réglementaires.

Les doctorats qui traitent de la déviance juvénile sont en définitive peu marqués par le champ disciplinaire dans lequel ils s'inscrivent. Les "docteurs ès délinquance juvénile" utilisent les mêmes mots, quelle que soit la faculté dont ils dépendent. Les termes médicaux abondent dans les mémoires juridiques et les expressions juridiques émaillent les dissertations médicales. L'analyse lexicologique des titres permet cependant de relever quelques écarts.

\section{I-2 Les mots pour le dire}

La désignation du sujet de la recherche doctorale peut prendre deux formes, parfois associées, dans des titres et sous-titres qui se veulent explicites et sont donc généralement assez longs. Dans la première, un problème est désigné par un substantif - la protection, la délinquance, la criminalité, le meurtre... - puis complété par un adjectif qualificatif ou un complément déterminatif - juvénile, infantile, chez l'enfant... - qui renvoie au jeune âge des individus concernés. Dans la seconde, la population objet de l'étude est nommée - la jeunesse, l'enfance, les mineurs... - puis elle est qualifiée de délinquante, coupable, irrégulière, etc. Nous nous sommes simplement attaché à mesurer la fréquence des substantifs qui servent à désigner le mineur de justice et celle des adjectifs qui le qualifient. 
Lorsqu'il s'agit de donner un nom à la population étudiée, les mots enfance et enfant(s) arrivent largement en tête, tant en lettres et droit (8 occurences) qu'en médecine (16 occurences). Le mot mineur (et ses dérivés féminin ou pluriel) vient en seconde position, là encore chez les juristes (4 occurences) comme chez les médecins (6 occurences). Si, dans la première forme syntactique évoquée plus haut, la délinquance est généralement qualifiée de juvénile, jeunesse n'apparaît qu'une fois et jeune jamais. Par contre, la notion d'adolescent est utilisée, au niveau des titres, par les seuls médecins (3 occurences). Faut-il y voir le poids du champ médico-psychologique dans l'effort de définition et de construction du concept même d'adolescence ? ${ }^{(14)}$

Notre corpus traitant de l'enfant de justice, celui qui relève des tribunaux, et non de l'enfance déficiente ou inadaptée en général, ${ }^{(15)}$ le qualificatif de délinquant/délinquante ne pouvait que dominer ( 9 occurences au total), d'autant que la loi du 27 juillet 1942, qui entend réorganiser la Justice des mineurs, utilise dans son titre l'expression enfance délinquante. La locution enfance coupable, avec 2 occurences chez les juristes et 3 chez les médecins, survit assez bien au XIXème siècle qui l'a vu naître. Exception faite du mineur pervers de constitution déjà cité et des enfants abandonnés, ${ }^{(16)}$ les lettres et le droit s'en tiennent à ce court registre lexical. Il n'en est pas de même du côté de la médecine. Le vocabulaire des thérapeutes est beaucoup plus étendu. Ils utilisent notamment tous les qualificatifs de la neuropsychiatrie infantile, comme déficiente, anormal, ${ }^{(17)}$ irrégulière... Peut-on voir là l'écho des débats du Conseil technique de l'enfance déficiente et en danger moral créé en 1943, où les psychiatres comme Georges Heuyer, Daniel Lagache, Robert Lafon occupent le premier rang ? Remarquons simplement que le terme générique retenu par le Conseil technique, enfance inadaptée, ne séduit pas nos auteurs. ${ }^{(18)}$

\section{II) ÉTIOLOGIE, NOSOGRAPHIE, TRAITEMENT}

Dans les travaux que nous avons pu lire, la déviance des mineurs génère trois niveaux de discours. Nos auteurs s'interrogent sur les causes de la
1914. HEUYER fut chef de clinique dans le service de DUPRÉ.

(13) Georges-

Dominique PESLE,

L'Enfance délinquante, vue d'un centre de triage, Paris, OTI, 1945, $67 \mathrm{p}$

(14) Gabrielle

HOUBRE,

"Adolescence, adolescent, adolescente au 19 ème siècle", in Adolescence nouvelle folie? IVème journée d'études cliniques de l'Union pour la défense de la santé mentale (UDSM), Fontenay-sousBois, 1996, pp. 8-23 ; François MARTY (dir.) L'adolescence dans l'bistoire de la psychanalyse, repères, Paris, les Cahiers du collège international de l'adolescence, 1996, 279 p. ; Agnès THIERCÉ, Histoire de l'adolescence (1850-1914), Paris, Belin, 334 p.

(15) Christian ROSSSIGNOL, "Quelques éléments pour l'histoire du Conseil technique de l'enfance déficiente et en 
danger moral. Approche sociolinguistique et historique", le Temps de l'histoire, $\mathrm{n}^{\circ} 1$, février 1999, p. 21-39.

(16) DOUMERC (Melle), Des enfants abandonnés et de leur condition juridique, Toulouse, 1945.

(17) Anormal est en fait utilisé une fois comme substantif dans Gisèle ETIENNEY, Enfance déficiente en Côted'Or et assistance aux anormaux, Lyon, 1944, et une fois comme qualificatif dans Georges GROSSE, Protection médico-légale des enfants anormaux criminels, Paris, 1940.

(18) Sur les enjeux de la dénomination et sur la signification du choix d'inadapté, voir Christian ROSSIGNOL, Inadaptation, handicap, invalidation? Histoire et étude critique des notions, de la terminologie et des pratiques dans le champ professionnel de l'éducation délinquance juvénile, sur son étiologie pour reprendre leur expression favorite. Ensuite, à l'exception de l'avocat chilien Carrasco-Barrois, qui s'intéresse exclusivement aux Théories sur les causes de la criminalité infantile, ${ }^{(1)}$ tous s'efforcent de classer les enfants de justice, de construire des nosographies plus ou moins complexes, qui permettent de ranger chaque garçon ou fille dans une case bien déterminée. Enfin, brièvement dans les thèses de médecine, plus longuement dans celles de droit, ${ }^{(20)}$ les rédacteurs suggèrent des solutions "prophylactiques" et "thérapeutiques".

\section{II-1 Une étiologique éclectique}

A l'exemple du docteur Marrec, qui affirme : "Depuis plus de quarante ans en France la notion de criminel-né est périmé ${ }^{(12)}$ lorsqu'il se penche sur les causes de la délinquance juvénile, tous nos auteurs s'entendent pour rejeter la conception "anthropologique" de Cesare Lombroso. Les théories purement sociologiques qui attribuent la déviance - juvénile ou non - aux conditions sociales sont elles aussi délaissées, sauf par Mario CarrascoBarrois qui se distingue encore une fois en écrivant dans sa conclusion :

"Nous sommes entraînés à conclure que ce sont les facteurs sociaux les vraies causes de la criminalité infantile, comme de la criminalité en général. Ils agissent non seulement en donnant aux délinquants l'occasion de l'acte délictueux, et à leur personnalité morale une orientation antisociale, mais ils contribuent aussi, puissamment, à la naissance de la criminalité pathologique. " ${ }^{(22)}$

La quasi totalité de nos spécialistes pourrait finalement se reconnaitre dans les propos du docteur Gamet :

"Nous concevons plus facilement la réalité d'une théorie éclectique en matière d'étiologie de l'enfance coupable. Il nous semble que la délinquance des mineurs est l'aboutissement d'un triple courant familial, social et individuel. L'enfant naît avec un certain bagage de troubles caractériels liés à son hérédité. [...] La famille et la société à leur tour, $[. .$.$] graveront leur sceau, [...] et modifieront le deve-$ nir de l'enfant vers l'adaptation à la vie normale dans la collectivité, ou au contraire vers le conflit social. ${ }^{(23)}$

Cet éclectisme, le mot est aussi utilisé par Guy Rey dans l'introduction de son travail ${ }^{(24)}$ débouche sur des inventaires à la Prévert, où le social voisine avec le pathologique, la maturité pubertaire avec le quo- 
tient intellectuel, l'alcoolisme parental avec le défaut de formation professionnelle. Les auteurs essaient de regrouper les éléments de leurs énumérations dans des sous-ensembles dont le contenu varie d'un texte à l'autre. Joubrel distingue les causes physiques (maladies somatiques, comme les encéphalites) et les causes psychiques, qu'il divise en héréditaires et sociales. ${ }^{(25)}$ Le docteur Gamet classe l'hérédité tuberculeuse dans les facteurs familiaux, ${ }^{(2)}$ alors que son collègue Vanhems la range dans les "tares pathologiques héréditaires ". ${ }^{(27)} \mathrm{Ce}$ dernier, dans sa conclusion, positionne l'alcoolisme des parents parmi les causes sociales..., avant d'en faire quelques lignes plus loin un facteur héréditaire. ${ }^{(28)}$ Nous pouvons malgré tout dégager les grandes lignes de l'analyse causale de la délinquance juvénile, telle que les élèves des facultés de lettres, de droit ou de médecine les perçoivent.

\section{II-1-1 Quand le cinéma est plus menaçant que le chômage (Une appréhension particulière des causes dites sociales)}

Qu'ils soient médecins ou juristes, nos doctorant(e)s ne rejettent pas l'existence de facteurs sociaux dans la délinquance juvénile. Certes, comme nous l'avons vu, ils ne mettent pas tous la même chose sous l'étiquette "social" ; mais l'essentiel n'est pas le classement de telle ou telle observation sous telle ou telle rubrique. Plus significatif est, nous semble-t-il, la faible place, voire l'absence totale, d'une réflexion sur les classes sociales, les conditions de travail, la pauvreté, la misère, le chômage, dans les analyses "sociologiques" de nos auteurs.

Du côté des médecins, seuls Guy Rey et Pierre Flot se penchent succinctement sur l'origine socioprofessionnelle des parents :

"Il nous a paru intéressant de rechercher la participation de chaque classe sociale (étant entendu qu'il s'agit de la profession des parents et non de celle des enfants). Et remarquant au passage que la "clientèle" étrangère figure dans l'bonorable proportion de 20\%, nous avons trouvé 58 familles d'ouvriers (soit près de 40\%), 19 employés, 9 commerçants, 9 forains, 1 agriculteur, 1 profession libérale et 53 (soit plus d'un tiers) sans profession reconnue. " ${ }^{(29)}$

«Le milieu familial est le plus souvent modeste. Les parents sont petits cultivateurs, marins pêcheurs, ouvriers... " ${ }^{(30)}$ spéciale, Thèse Strasbourg I, 1999, pp. 141-143.

(19) Mario CARRAS-

CO-BARROIS, op. cit.

(20) Voir par exemple

la thèse de Fernand

JOUBREL, L'Enfance

coupable (plan de réforme

de la législation et des

institutions existantes),

Saint-Brieuc (Faculté de

Rennes), Imprimerie

moderne, 1942, 214 p.

(21) Jean MARREC,

Homicide volontaire chez

l'enfant. Contribution à

l'étude de l'enfance

coupable, Lyon, Bosc

Frères \& L. Riou, 1943, (n.p.).

(22) Mario CARRAS-

CO-BARROIS, op. cit., p. 151.

(23) André GAMET, Contribution à l'étude de l'enfance coupable. Les facteurs familiaux et sociaux (A propos de la cartographie lyonnaise de la délinquance juvénile), Lyon, Emmanuel Vitte, 1941, pp. 21-22.

(24) Guy REY, Mineurs délinquants 
récidivistes. Contribution à l'étude de l'enfance coupable, Lyon, Bosc Frères \& L. Riou, 1943, p. 13.

(25) Fernand JOUBREL, op. cit., pp. 36-39.

(26) André GAMET, op. cit., pp. 39-40.

(27) René VANHEMS, Étude statistique portant sur 1.150 observations de mineurs délinquants réunis au Centre de triage de la Faculté de médecine de Lyon, Lyon, Imprimerie des Facultés, 1939, pp. 52-53.

(28) Ibid., p. 115.

(29) Guy REY, op. cit., p. 22.

(30) Pierre FLOT, Constatations médicales et sociales relatives à la délinquance juvénile en Bretagne, Paris, R. Foulon, 1945, p. 22.

(31) Philippe REYHERME, op. cit. p. 46.

(32) Ibid., pp. 49-50.

(33) Ibid., p. 50.

(34) Ibid., p. 46.
L'abbé Rey-Herme n'est guère plus prolixe. Il se contente d'affirmer, sans indiquer de sources, que « $80 \%$ des jeunes délinquants appartiennent à des milieux ouvriers ou commerçants, et $4 \%$ seulement à des familles exerçant une profession libérale. " ${ }^{(31)}$

Ajoutons que, dans les études de cas qui illustrent les propos généraux, les observateurs n'oublient jamais d'indiquer que le père (ou la mère) est éthylique ou épileptique, mais nous disent rarement quel emploi il (elle) occupe et s'il (si elle) est au chômage.

Les conditions de travail ne sont guère mieux traitées que les CSP. Philippe Rey-Herme, dans un chapitre sur les "données actuelles des disciplines annexes capables de préciser le problème ", consacre deux pages au " milieu scolaire et de travail ». ${ }^{(32)}$ Son analyse des conditions de travail se réduit à une remarque sur les moqueries dont les apprentis peuvent être victimes de la part de leurs aînés..$^{(3)}$ Une ou deux notations sur " l'absence aujourd'hui fréquente de la mère retenue loin du foyer par le souci de consolider un budget en équilibre toujours instable $»^{(34)}$ complète ce très bref panorama. ${ }^{(35)}$

La pauvreté et la misère ne sont pas totalement laissées dans l'ombre. Les mauvaises conditions de logement sont même assez unanimement dénoncées. "Le taudis rend toute vie familiale impossible, chacun aura envie de fuir le foyer si peu confortable, d'où l'instabilité familiale ", écrit par exemple René Vanhems, ${ }^{\left({ }^{(6)}\right)}$ en accord sur ce point avec tous nos auteurs. Le mot chômage n'apparaît que dans le texte d'André Gamet et, quand l'absence de travail est mentionnée, pour les mineurs comme pour leurs parents, elle est "psychologisée", interprétée en tant que signe de paresse ou d'aboulie. De même le turn-over ne saurait être autre chose que la manifestation d'une instabilité psychique structurelle. ${ }^{(3)}$

L'organisation socio-économique est en définitive très peu interrogée. On consacre beaucoup plus de lignes à l'analyse des effets (supposés) criminogènes du cinéma ${ }^{(38)}$ qu'à l'étude de l'influence des conditions matérielles d'existence. Le plus "sociologue" de nos docteurs, André Gamet, rédige 32 lignes sur les effets de la misère et du chômage et 137 lignes agrémentées d'un tableau statistique sur la nocivité du septième art. Il faut dire que la contestation politico-sociale est décrite en 1943, par l'un des maîtres à penser du problème de l'enfance malheureuse, 
comme une pathologie :

"L’bygiène mentale doit agir dès la naissance et même avant. [...] Sinon, des conséquences graves en découleraient. Elles vont depuis les simples irrégularités scolaires et familiales et les mauvais rendements intellectuels et professionnels, si fréquents, jusqu'à l'hostilité ouverte envers la société, et dont la délinquance est la manifestation individuelle essentielle, et l'agitation sociale la traduction collective principale. " ${ }^{(39)}$

Nous comprenons mieux, quand l'agitation sociale, - faut-il lire Front Populaire ou Résistance ? - devient un problème d'bygiène mentale, pourquoi les facteurs sociaux sont rabattus sur la responsabilité individuelle ou réduits à l'anecdotique. Voilà comment le cinéma devient, après le théâtre et la lanterne magique et avant la télévision, une raison majeure du développement de la criminalité infantile.

En définitive, la "reine" des causes sociales, celle qu'aucun de nos auteurs n'oublie de mentionner, est la dissociation familiale. Avant de traiter cet aspect particulier qui mérite un développement autonome, nous examinerons le poids de l'héréditarisme dans les discours étiologiques des "années noires".

\section{II-1-2 Le poids de l'héréditarisme}

A l'exemple de Louis Bagon, les observateurs recherchent systématiquement les antécédents héréditaires auxquels ils attribuent une partie du caractère et du comportement du jeune délinquant dont ils retracent le parcours : "Le mineur a une lourde bérédité. Le père alcoolique a été condamné 7 fois pour des motifs très variés. Un oncle du père a été atteint de troubles nerveux. [...] C'est très vraisemblablement un hérédo-syphilitique. [...] La mère a eu plusieurs fausses couches et 2 enfants prématurés. " ${ }^{(40)}$ Nous comprenons aisément à la lecture de cet exemple que l'hérédité dont il est question n’a guère à voir avec la génétique moderne. A cette époque, la transmission héréditaire de caractères acquis n'est pas plus invraisemblable que l'existence d'un génome stable. Qu'un rachitique victime d'un climat délétère et d'une mauvaise alimentation puisse engendrer un autre rachitique est une idée assez largement partagée. Cependant, nous voyons que l'usage du concept d'hérédité est poussé beaucoup plus loin. Quand on

Voir aussi Guy REY, op.

cit., p. 21

(35) Sur ce thème,

voir Christian

LÉOMANT, "Travail de

la femme et délinquance

juvénile. Essai de présen-

tation des conditions de

production d'un fait

social", Annales de

Vaucresson, $\mathrm{n}^{\circ} 13,1975$,

pp. 111-125.

(36) René VAN-

HEMS, op. cit., p. 22.

(37) Sur ce point, voir Jean-Jacques YVOREL,

"De Gavroche aux

apaches, sources et

méthode d'une histoire

des illégalismes juvé-

niles”, in Frédéric

CHAUVAUD, Jacques-

Guy PETIT, (dir),

L'histoire contemporaine et les usages des archives

judiciaires (1800-1939),

Paris, Honoré Cham-

pion, 1998, pp. 457-458.

(38) Sur les 21 textes

que nous avons lus, 16 consacrent un développement aux effets pervers du cinéma.

(39) Robert LAFON, 
"Les bases d'une psychologie médicale pratique", conférence aux journées régionales de l'enfance malheureuse, avril 1943, cité par Michel CHAUVIÈRE, Enfance inadaptée : l'héritage de Vichy, Paris, les Editions ouvrières, 1980, p. 78.

(40) Louis BEGON, op. cit., p. 24-25.

(41) Par exemple René VANHEMS, op. cit., p. 52-53 et Émile CHAUVEL, op. cit., p.16.

(42) Augustin Bénédict MOREL, Traité de la dégénérescence physique, intellectuelle et morale de l'espèce bumaine et des causes qui produisent ces variétés maladives, Paris, 1857, 750 p.

(43) André GAMET, op. cit, p. 21.

(44) André PER-

REAU, op. cit., p. 39-40.

(45) Tous les médecins qui ont effectué un stage dans un centre de tri ou une maison d'arrêt donnent leur pourcentage de dissociation toujours explique la délinquance du fils par la tuberculose de l'un des parents, ${ }^{(41)}$ ce n'est plus une tare spécifique qui est transmise, mais un quantum de dégénérescence, une part de son essence. Ainsi, notre rachitique peut donner naissance à un hystérique, un alcoolique, un morphinomane... ou un assassin, qui seront ce qu'ils sont parce que fils ou fille de rachitique. Voilà qui permet de passer du registre du pathologique au registre du comportement social (alcoolisme, toxicomanie, délinquance, etc.) ; dans tous les cas de figures, cette transduction fournit une étiologie génétique. Cette théorie, mise au point au milieu du XIXème siècle par l'aliéniste Augustin Bénédict Morel, ${ }^{(42)}$ permet d'expliquer aisément que les mêmes causes produisent des effets différents, tout en conservant à l'hérédité son caractère d'universalité.

Cet héréditarisme sert aussi d'arme contre la psychanalyse, dont les succès croissants risquent d'empiéter sur le domaine encore fragile de la pédopsychiatrie, et excuse par avance tous les échecs de la prise en charge :

"Si les perversions morales de l'enfant sont acquises et déterminées par des conflits affectifs, une éducation bien comprise peut éviter l'éclosion des anomalies morales. D’autre part, celles-ci, une fois acquises, mais indépendantes de la constitution de l'individu, peuvent être guéries par l'analyse. Tel est le verdict des psychanalystes. Pour Henyer et ses élèves, au contraire, chaque fois que la déviation du caractère ou du sens moral est un facteur héréditaire ou constitutionnel, le même essai éducatif sera bien aléatoire quant à ses résultats. " ${ }^{(43)}$

Si le primat de l'hérédité dans l'étiologie de la délinquance ne fait aucun doute pour les médecins, les juristes, comme Perreau, sont plus circonspects. Dans sa thèse consacrée aux "pervers de constitution", il doute parfois de l'innéité de cette psychopathie, même s'il reconnaît " l'indissociabilité des notions d'hérédité et d'éducation " :

"C'est pourquoi nous critiquons le terme même de "pervers de constitution", en ce sens que nous ne pensons pas qu'il y ait là un instinct inné. C'est avec l'achèvement de la formation du caractère que se fixent les tendances. ${ }^{(4)}$

La transmission intergénérationnelle des tares constitutionnelles à l'origine des déviances infanto-juvéniles étant généralement admise, comment nos auteurs articulent-ils cette hérédo-génèse avec un grand facteur social : la dissociation familiale? 


\section{II-1-3 La dissociation familiale : première cause de la délinquance juvénile?}

S'il est un point sur lequel tous nos auteurs sont en accord, c'est bien sur l'influence de la dissociation familiale. A grand renfort de statistiques, celles qu'ils produisent ${ }^{(45)}$ et celles qu'ils rappellent, ${ }^{(46)}$ ils postulent unanimement à un lien de causalité entre la dissociation du couple parental et la délinquance des enfants.

Le "concept" de dissociation est très large et englobe toutes les structures familiales qui s'écartent peu ou prou du modèle dominant, à savoir la famille conjugale, légalement constituée, dont tous les membres sont en vie. Guy Rey inclus même le travail salarié des parents (lire des femmes) dans les facteurs de dissociation :

"Combien voyons-nous de milieux dissociés par le veuvage, la séparation, le divorce, le remariage, le concubinage, enfin le travail des parents les soustrayant de la maison pour la journée entière, tous ces facteurs qui font que l'enfant préfere la rue au foyer où il est délaissé. " ${ }^{(47)}$

Nous n'allons pas reprendre ici la question de la construction et de la déconstruction de cette catégorie largement traitée par ailleurs, même si le stéréotype fonctionne toujours. ${ }^{(48)}$ Relevons seulement que l'action délétère des familles anormales (le terme est utilisé par nos auteurs) est souvent stipulée par le fait même de la corrélation statistique (puisque 60\% des mineurs délinquants ont une famille désunie ou amorale, la désunion ou l'amoralité familiale est une cause de la délinquance). Les systèmes explicatifs les plus élaborés font appel au paradigme éco-sociologique de "l'imitation", du "mauvais exemple", de "la rue". Contrairement à Georges Heuyer qui cherche à construire un système cohérent articulant dissociation familiale et hérédité, ${ }^{(49)}$ nos rédacteurs juxtaposent simplement les deux "causalités".

\section{II-2 Une nosographie eugéniste?}

L'activité taxinomique n'est pas nouvelle. Elle connaît à la fin du XIXème siècle un développement considérable. Cet acharnement classificateur concerne particulièrement les enfants, qui constituent « une sous-population soumise depuis longtemps à un processus très raffiné de délimitation ". ${ }^{(50)} \mathrm{La}$ supérieur ou égal à 50\%.

(46) Outre les chiffres

de l'incontournable

Georges HEUYER,

l'étude du criminologue lyonnais M. RAUX

(1890) et les statistiques d'Henry JOLY sont fréquemment citées.

(47) Guy REY, op. cit., p. 21.

(48) Sur ce point, voir Nadine LEFAU-

CHEUR, Dissociation

familiale et délinquance

juvénile, les avatars scientifiques d'une représentation sociale, Paris, Rapport pour la CNAF (200 p. ronéo), et Christian LÉOMANT,

"Dissociation familiale et délinquance juvénile. Remise en cause d'un stéréotype", Annales de Vaucresson, $\mathrm{n}^{\circ} 12,1974$, pp. 119-139.

(49) Nadine LEFAUCHEUR, Psychiatrie...”, art. cit., pp. 328-329.

(50) Olivier FAURE, Sylvie DAD, Françoise TETARD, "La volonté de classer”, les Cabiers de 
la recherche sur le travail social, $\mathrm{n}^{\circ} 19,1990$, p. 10.

(51) Jean MARREC,

Homicide volontaire chez l'enfant. Contribution à l'étude de l'enfance coupable, Lyon, Bosc Frères \& L. Riou, 1943, (n.p.), observation $\mathrm{n}^{\circ}$ XVI.

(52) Daniel

LAGACHE, Procès verbal de la réunion du Conseil technique de l'enfance déficiente et en danger moral du 30 mars 1944, cité par Christian ROSSIGNOL, op. cit., p. 143.
Seconde guerre mondiale ne freine pas vraiment, dans ce domaine, la production de catégories. Comme nous l'avons déjà dit, ce ne sont généralement pas les impétrants docteurs qui révolutionnent la science et inventent donc les nouvelles grilles de classement, mais ils rendent compte des débats en cours. A la lecture du corpus que nous avons constitué, nous voyons immédiatement que la catégorisation est l'œuvre des médecins ou plus précisément des psychiatres. Les juristes ou les "littéraires", qui sont à l'origine de bien des dénominations comme enfance coupable, malheureuse, moralement abandonnée, mineurs vicieux, etc., ne produisent plus de nouvelles sous-divisions ni de nouveaux intitulés génériques et semblent se rallier à la "science positive" des neuropsychiatres. Les thèses, toutes disciplines confondues, exposent donc les nosographies réalisées par les pédopsychiatres ou en utilisent certains éléments.

La lecture continue des situations particulières de mineurs qui émaillent les propos généraux permet de saisir la nature et de comprendre la construction des catégories nosographiques. La présentation de ces "cas cliniques" commence par un résumé des éléments de type étiologique (moralité de la famille, antécédents médico-psycho-sociaux de la parentèle et du mineur, parcours scolaire et professionnel, description de l'habitat). Viennent ensuite des informations sur le caractère, le comportement du mineur et les résultats des tests qu'il a subis. La conclusion s'appuie uniquement sur cette dernière partie symptomatique et prend la forme d'un classement associé à une proposition : " Il s'agit d'un sujet amoral, anémotif, inaffectif. En somme, il s'agit d'un pervers et plus tard contagieux. Il est à placer dans une maison d'éducation surveillée. " ${ }^{(51)}$ Nous retrouvons là cette double nosographie qui sera systématisée en 1944 par le Conseil technique de l'enfance déficiente et en danger moral avec, d'un côté, une classification dite "médico-psychologique" et, de l'autre, une classification dite "médico-pédagogique". La première adoptera, nous dit le professeur Lagache, "un point de vue aussi descriptif que possible, en évitant de faire intervenir les théories et les doctrines ; c'està-dire que la symptomatologie doit jouer un rôle plus important que l'étiologie et la pathogénie ". ${ }^{(52)}$ La seconde est établie, toujours selon Daniel Lagache, 
" du point de vue de l'assistance et du traitement dont les enfants relèvent » et indique la destination des mineurs en fonction de leur place dans la nosographie médico-psychologique. Notons que la liste des solutions est singulièrement réduite : si la première taxinomie comporte plus de 20 pages, la classification médico-pédagogique tient en une demi-page. ${ }^{(53)}$ Elle consiste surtout à répartir la population en trois grandes catégories : les éducables, les semi-éducables et les inéducables. La procédure qui consiste in fine à mesurer la dangerosité présumée du sujet sans prendre en compte la cause des troubles et à préconiser la solution la plus adaptée à la protection de la société relève bien d'une forme de défense sociale, d'eugénisme plus ou moins radical. La forme de la transgression prend finalement une importance considérable et le mineur récidiviste ${ }^{(54)}$ ou criminel ${ }^{(55)}$ a peu de chance d'échapper à la catégorie la plus grave : le pervers constitutionnel.

L'imprégnation eugénique de la période est encore plus nette quand on se penche sur les solutions préconisées par nos auteurs.

\section{II-3 Le traitement entre pouvoir médical et tentation eugénique}

Les solutions proposées par nos auteurs peuvent être rangées sous diverses rubriques. Viennent d'abord des propositions très générales, comme de "réformer la censure en matière de cinéma, de théâtre et de littérature " ${ }^{(56)}$ ou de renforcer "les lois contre le divorce ". ${ }^{(57)}$ Arrivent ensuite des plans de réorganisation du dispositif de prise en charge de l'enfance irrégulière. L'esprit de la réforme est assez bien résumé par GeorgesDominique Pesle, élève d'Heuyer : "La coordination des divers pouvoirs judiciaire, pédagogique, médical, social est indispensable. " ${ }^{(58)}$ Nos rédacteurs proposent généralement la création d'un dispositif unifié de prise en charge de l'enfance malheureuse et l'édification d'un code de l'enfance. Tous militent pour la spécialisation des magistrats de la jeunesse. Quant aux méthodes de rééducation, pour Joubrel, pour Rey-Herme, pour Pesle, elles peuvent se résumer en une phrase : "utilisation au maximum de la méthode scoute". Mais, surtout, l'immense majorité de nos docteurs en droit ou en médecine insistent sur le rôle fondamental du dépistage et se félicitent du développement des centres de triage et d'observation insti-
(53) Ibid., p. 144.

(54) Guy REY,

Mineurs délinquants

récidivistes. Contribution à

l'étude de l'enfance

coupable, Lyon, Bosc

Frères \& L. Riou, 1943, $64 \mathrm{p}$.

(55) Jean MARREC,

Homicide volontaire chez

l'enfant. Contribution à

l'étude de l'enfance

coupable, Lyon, Bosc

Frères \& L. Riou, 1943,

64 p., et Louis BEGON,

Etude du meurtre chez

l'enfant et chez l'adolescent,

Paris, E. Le François,

1940, 32 p.

(56) André GAMET,

op. cit., p. 120.

(57) Ibid., p.117.

(58) Georges-

Dominique PESLE, op. cit., p. 62. Le pouvoir central s'efforcera de mettre en place cette coordination en créant notamment les

Associations régionales de sauvegarde de l'enfance et de l'adolescence (ARSEA). Voir Michel CHAUVIÈRE, op. cit. 
(59) Geneviève

MAZO, Le Centre d'observation et la Loi $d u$ 27 juillet 1942 relative à l'enfance délinquante, Paris, H. Van Etten, 1944, 215 p.

(60) Sur l'eugénisme et le frein que représente le catholicisme, voir Anne CAROL, Histoire de l'eugénisme en France: les médecins et la procréation, XIX-XXème siècle, Paris, le Seuil, 1995, 381 p.

(61) Philippe REY-

HERME, op. cit., p. 59

(62) Jean MARREC, op. cit., p. 68

(63) Guy REY, op. cit., p. 61 .

(64) André GAMET,

op. cit., pp. 121-122.

C'est Gamet qui souligne. tués par la loi du 27 juillet $1942 .{ }^{(59)}$ Consciemment ou non, cela revient, d'une part, à confier l'essentiel du pouvoir décisionnel aux médecins et plus particulièrement aux neuropsychiatres et, d'autre part, à exclure les enfants classés pervers constitutionnels. Il s'agit avant tout de repérer ces derniers, que tous nos auteurs, à l'exception de Carrasco-Barrois, considèrent comme inéducables.

\section{II-3-1 L'exclusion des inéducables}

Philippe Rey-Herme, prêtre catholique, peut difficilement être soupçonné de verser dans l'eugénisme radical et matérialiste d'un Alexis Carrel ${ }^{\left({ }^{(0)}\right.}$; il écrit pourtant à propos des enfants classés constitutionnellement pervers : "On doit en général se contenter de les mettre dans l'impossibilité de nuire, sans pouvoir espérer jamais les rendre à une vie à peu près normale ». ${ }^{(61)}$ L'opinion de notre philosophe est partagée par tous les médecins qui, à l'exemple du docteur Marrec, affirment que "les seules mesures qui se justifient consistent à placer l'enfant dans des conditions telles qu'il lui sera impossible de nuire à la société ». ${ }^{(62)}$ Selon le docteur Guy Rey, qui adopte en la matière les idées de ses confrères, cette mise à l'écart doit s'appuyer sur un dépistage précoce : "Nous devons nous attacher à les [les pervers] dépister le plus tôt possible, afin de les isoler et de les soustraire à la collectivité, comme l'on fait pour les malades contagieux. " ${ }^{(63)} \mathrm{Ce}$ double programme d'étiquetage/exclusion reçoit l'agrément du docteur Gamet, qui le formule avec plus de brutalité langagière : "L'édifice de préservation de l'enfance ne peut être entièrement bâti que s'il comprend des mesures de dépistage précoce des pervers contagieux. [...] Tous les suspects dépistés seront examinés par des médecins spécialisés en neuro-psychiatrie infantile et en toutes questions relatives à l'enfance coupable. - La sanction sera l'élimination formelle de tous les individus contagieux, pervers constitutionnels et pervertis. - Il ne peut être question ici de faire preuve de sensibilité. " ${ }^{(64)}$ André Gamet, ancien externe des hôpitaux de Lyon, élève de l'École du service de santé militaire et diplômé de médecine légale et de psychiatrie, ne nous indique pas comment il entend procéder pour éliminer " tous les individus contagieux, pervers constitutionnels et pervertis ». Il n'est pas le seul à rester silencieux sur la méthode. Le docteur Marrec, lui aussi élève de l’École du service de santé militaire et 
ancien externe des hôpitaux de Lyon, est plus précis : «Il faut substituer à la notion métaphysique et arbitraire de la responsabilité la notion objective de la nocivité du criminel, de la témébilité [sic] du pervers et créer des établissements spéciaux intermédiaires à l'asile et à la prison, des asiles de sécurité pour les anormaux dangereux, les psychopathes vicieux, les imbéciles moraux, dangereux, difficiles, etc., en un mot pour les sujets atteints de perversions instinctives. ${ }^{(65)}$

Pour les gens de droit, ce programme se heurte à un obstacle juridique : "Cette absence de reconnaissance légale [du pervers] fait aussi que son dépistage clinique ne peut être qu'une indication à veiller, mais non à agir. " ${ }^{(6)}$ Notre juriste, André Perreau, propose alors d'introduire une distinction légale entre "mineurs corrigibles" et "mineurs inamendables", ${ }^{(67)}$ la séparation des deux groupes étant l'œuvre de centres d'observation et de triage où officient des neuropsychiatres. Dès lors, "l'asile de sûreté [...] assurera l'élimination des pervers et [...] deviendra un des centres de protection de la Société contre les inamendables et les incorrigibles ". ${ }^{(6)}$ Dernier point et non le moindre, ce "traitement", qui se traduit par un enfermement de très longue durée, voire définitif, peut s'appliquer à un enfant préventivement, avant qu'il ait commis un crime ou un délit.

Pour Vanhems, 5\% des 1150 mineurs délinquants réunis au centre de triage de la Faculté de médecine de Lyon appartiendraient à la dangereuse catégorie des pervers. ${ }^{(6)}$ Au centre d'accueil d'Ille-et-Vilaine, le docteur Pierre Flot découvre 7,5\% d'amoraux pervers chez les garçons et $21 \%$ chez les filles. ${ }^{(7)}$ Les tribunaux pour enfants et adolescents traitent environ 50.000 affaires par an ${ }^{(7)}$; si le programme eugénique préconisé par nos "thésards" et par leurs maîtres avait été mis en place, c'est rapidement plusieurs dizaines de milliers de places d'asile de sûreté qu'il eût fallu construire!

\section{III) “SCIENCE NAZIE” ET RÉFLEXION FRANÇAISE SUR LA DÉLINQUANCE JUVÉNILE}

Si nous trouvons chez Gamet quelques propos xénophobes sur la colonie étrangère "source de contamination pour les mineurs autocbtones ", ${ }^{(2)}$ c'est la seule trace de racisme déclaré, et notre corpus ne révèle aucune marque
(65) Jean MARREC, op. cit., p. 63.

(66) André PER-

REAU, op. cit., p. 139.

(67) Ibid., p. 141.

(68) Ibid., p. 141.

(69) René VAN-

HEMS, op. cit., p. 116.

(70) Pierre FLOT, op. cit., p. 46.

(71) Voir, dans ce numéro, l'article de Vincent PEYRE.

(72) André GAMET, op. cit., p. 74 . 
(73) Fernand JOU-

BREL, op. cit., p. 187-192.

(74) André GAMET,

op. cit., p. 105-107.

Sur les Compagnons de

France, voir Pierre

GIOLITTO, Histoire de

la jeunesse sous Vichy,

Paris, Librairie acadé-

mique Perrin, 1991,

pp. 507-513.

(75) Sur le rôle et la responsabilité des scientifiques dans la mise en place de l'extermination des malades mentaux, handicapés, asociaux et bien sûr des Tziganes et des Juifs, voir Benno MÜLLER-HILL, Science nazie, science de mort. L'extermination des Juifs, des Tziganes et des malades mentaux de 1933 à 1945, Paris, Odile Jacob, 1989, 246 p.

(76) Théorie de l'hérédité humaine et hygiène raciale.

(77) C'est un véritable magistère qu'exerce Georges Heuyer ; il est cité par tous les auteurs que nous avons lus. d'antisémitisme. Bien sûr, les propos de Fernand Joubrel sur les chefs ${ }^{(73)}$ ne pas sont pas très éloignés des discours du maréchal sur le même thème, mais il est difficile de parler d'adhésion au fascisme. La marque la plus patente d'une certaine appétence pour l'idéologie nazie est lisible dans la thèse de Gamet précédemment citée. La trace est assez ténue. Notre médecin militaire reprend les critiques formulées par la presse collaborationniste contre les Compagnons de France. Ce mouvement de jeunesse, pétainiste mais opposé aussi bien au racisme et à l'antisémitisme qu'à la création d'un mouvement de jeunesse d'État unique, est accusé d'être un repère de délinquants et un lieu de contamination délictueuse. ${ }^{(74)}$

La teneur de certains propos ne doit pas nous tromper : sur le plan scientifique, nos auteurs ne sont pas, à une exception près sur laquelle nous reviendrons, influencés directement par les théories professées en Allemagne par les animateurs de l'Institut de l'empereur Guillaume d'anthropologie, de théorie héréditaire et d'eugénisme. ${ }^{(75)}$ Aucun ne mentionne l'ouvrage, jugé fondamental par Hitler, de Baur, Fischer et Lendz, Menschliche Erblichkeitslehre und Rassenbygiene ${ }^{(76)}$ ou les écrits de Heyde, le psychiatre, "père" de l'euthanasie des malades mentaux. Nos docteurs ès délinquance juvénile citent Georges Heuyer, ${ }^{(77)}$ Etienne Martin ou Pierre Mazel, ses homologues lyonnais, le docteur Male qui a soutenu en 1927 une thèse sur Le rôle de l'hérédo-syphilis dans l'étiologie des troubles mentaux de l'enfance, mais aussi Louis Albanel ou Henry Joly, juristes qui écrivaient sur le sujet dans les années 1900. Leurs références sont donc françaises et laissent de côté les eugénistes les plus radicaux de l'hexagone, tels Charles Richet, Alexis Carrel ou Justin Godard. Les analyses qu'ils produisent, les solutions qu'il proposent ne sont pas originales, mais directement issues de cette littérature spécialisée des années d'avant-guerre.

Une thèse pourtant tranche avec la retenue (toute relative) majoritaire et défend la mise en place d'une politique prophylactique directement inspirée par les lois du Reich : celle de la juriste Françoise Liévois. Soutenue en 1944, mais imprimée en 1946, La délinquance juvénile, cure et prophylaxie ne fait pas partie de notre corpus initial. 


\section{III-1 Une "prophylaxie" radicale}

Dès l'introduction, Françoise Liévois soutient une position relevant d'un eugénisme radical peu répandu en France : «Au nom de cette liberté qui n'est qu'un mythe, elle [la France] laisse procréer et croître en son sein l'enfant du tuberculeux, du syphilitique, de l'alcoolique et du fou même. Elle n'ose attenter à l'intégrité physique de l'être dont il ne peut que sortir un danger ou une charge pour elle, mais elle envoie à la mort le plus beau, le meilleur de sa puissance génératrice ${ }^{(78)}$ Elle annonce ensuite le plan en deux parties de son travail : "La première examinera le régime juridique et pénitentiaire applicable à l'Enfance. [...] La deuxième partie de notre étude s'intitulera Les projets et les Espoirs : Projets de cure [...] Espoirs en une prophylaxie de la délinquance juvénile. [...] Cette seconde partie constituera notre thèse proprement dite, celle que nous soutenons. " ${ }^{(79)}$ C'est bien Outre-Rhin que madame Liévois va chercher le modèle sur lequel elle fonde tant d'espoirs : " Dans les droits modernes, le problème [de la prophylaxie] fut étudié en lui-même et résolu comme en Allemagne et en Italie, ou seulement abordé indirectement et réglé partiellement par des dispositions politiques, sociales ou économiques comme en Angleterre et aux États-Unis. Enfin, comme en France, il n'a pu être envisagé que tout récemment. ${ }^{\left({ }^{(8)}\right.}$ Elle vante les premières expériences américaines : "Ayant à se défendre contre une triple immigration parfois indésirable (Indiens, Jaunes, Noirs), il était logique que la grande République américaine prît, une des premières, de sévères mesures pour protéger sa descendance. ${ }^{(81)}$ Et elle revient sur l'exemple allemand, prix d'excellence en la matière : "C'est l'Allemagne qui était allée le plus loin en promulguant le 18 octobre 1935 la loi sur la santé du mariage complétée par les lois de Nuremberg essentiellement raciales. " ${ }^{(82)}$ Elle félicite nos voisins germaniques pour les 56.244 stérilisations réalisées durant l'année 1934, avant d'assurer : "Il ne s'agit pas quant à nous de faire du racisme, nous n'avons en effet aucune raison de supposer qu'un habitant de Stockbolm soit supérieur à un Madrilène ou inversement. Mais à l'intérieur d'une même race [...], il est avéré que des êtres sains ont plus de chance de créer des êtres normaux que les pauvres déchets d'bumanité qui s'accouplent au hasard des beuveries et dans l'ombre des taudis. ${ }^{\left({ }^{(3)}\right)}$ Elle stigmatise ceux qui osent prétendre que la stérilisation est « une méthode scientifiquement absurde» et "un crime odieux [...] contre la race bumaine toute entiè-
(78) Françoise LIÉ-

VOIS, La délinquance juvénile, cure et prophylaxie, Paris 1946, p. 7.

(79) Ibid., p. 10.

(80) Ibid., p. 127. C'est nous qui soulignons.

(81) Ibid.,. p. 128.

(82) Ibid., p. 128.

(83) Ibid., p. 130. 
(84) Ibid., p. 130.

(85) Max LAFONT,

L'extermination douce,

Rennes, AREFPPI, 1988,

256 p. Dans sa thèse,

LAFONT ne traite pas

de l'Alsace, directement

administrée par les auto-

rités allemandes.

L'extermination des

malades mentaux et

autres indésirables n'y

fut pas douce. re ", avant de s'exclamer : "Ce n'est pas un crime contre l'bumanité d'enfanter des monstres qui voleront, violeront, pilleront! ${ }^{(84)}$

\section{Conclusion}

S’ils ne basculent généralement pas dans l'eugénisme "dur", nos auteurs n'en proposent pas moins une mise à l'écart des "inéducables". Ce projet n'est pas neuf. Ses fondements intellectuels sont à chercher, d'une part, du côté de la théorie de la dégénérescence et plus globalement de la pensée héréditaro-eugéniste et, d'autre part, dans les préoccupations de la défense sociale nouvelle. Il prend, durant la période 40-45, un sens nouveau. Vouloir créer des asiles de sûreté pour enfants pervers quand les malades mentaux subissent une "extermination douce " ${ }^{(85)}$ n'est pas une proposition technique neutre. Dans la France occupée, l'exclusion avait toutes les "chances" de se transformer en extermination, "l'élimination formelle » en élimination réelle.

\section{THÈSES DE LETTRES, DE DROIT ET DE MÉDECINE PARUES EN FRANCE ENTRE 1939 ET 1945}

[Les titres précédés d'un astérisque "sont ceux des thèses auxquelles nous avons eu accès.]

\section{LETTRES}

CARRASCO-BARROIS Mario, *Théorie sur les causes de la criminalité infantile et juvénile, étude critique, Paris, Jouve, 1942, 158 p.

REY-HERME Philippe, *Quelques aspects du progrès pédagogique dans la rééducation de la jeunesse délinquante, Paris, Vrin, 1945, 190 p.

$\underline{\text { DROIT }}$

AUDIT Mme, Le problème de l'enfance délinquante, Bordeaux, 1944. 
BAJOTET Marc, *Les variations de l'incapacité du mineur en raison de son âge, Paris (Faculté de Dijon), 1941, 260 p.

CHASSOT Maurice, "Conséquences pénales et civiles de l'infraction commise par un mineur à la suite de la loi du 27 juillet 1942 sur l'enfance délinquante, Dijon, Darantière, 1943, 238 p.

DOUMERC Melle, Des enfants abandonnés et de leur condition juridique, Toulouse, 1945.

DUCAS Bernard, La délinquance juvénile. Recherche de solutions d'équité, Lyon, 1943.

JOUBREL Fernand, "L'Enfance coupable (plan de réforme de la législation et des institutions existantes), Saint-Brieuc (Faculté de Rennes), Imprimerie moderne, 1942, $214 \mathrm{p}$.

MAZO Geneviève, "Le Centre d'observation et la loi du 27 juillet 1942 relative à l'enfance délinquante, Paris, H. Van Etten, 1944, 215 p.

MONNET DE LORBEAU Mme, De la protection de l'enfance et de l'acte dit loi du 15 avril 1943, Toulouse, 1945.

PERREAU André, *Le mineur pervers de constitution, contribution à l'étude de l'enfance coupable, Lyon, Bosc Frères \& L. Riou, 1942, 152 p.

POULICARD Magdeleine, "L'assistance éducative, modifications apportées à l'article 2 de la loi du 24 juillet 1889 par le décret-loi de 1935, Trévoux (Faculté de Lyon), G. Patissier, 1941, 204 p.

ROUGIER Amédée, La protection pénale des mineurs, Avignon (Faculté de Montpellier), Barthélémie, 1945, 171 p.

WAQUET Pierre, "La protection de l'enfance. Étude critique de la législation et des sciences sociales, Rennes, Imprimerie réunies, 1942, $344 \mathrm{p}$.

\section{MÉDECINE}

BEGON Louis, *Etude du meurtre chez l'enfant et chez l'adolescent, Paris, E. Le François, 1940, 32 p.

BENCHARFI, Responsabilité atténuée. Quelques réflexions, Lyon, 1945.

BRETON Paul, *Considérations sur l'enfance délinquante à propos de 250 examens médico-psychologiques de mineurs détenus, Bordeaux, Delmas, 1943, $51 \mathrm{p}$. 
CHAUVEL Émile, "Contribution à l'étude médicale et sociale de la délinquance juvénile. Étude faite sur 109 mineurs du centre d'accueil de Rennes, Paris, 1945, 18 p. dactylographiées

DURY André, Hygiène mentale infantile. Les consultations de Pontarlier et Besançon depuis 1935, Lyon, 1944.

ETIENNEY Gisèle, Enfance déficiente en Côte-d'Or et assistance aux anormaux, Lyon, 1944.

FARGEAT Marie, Centres d'observation. Contribution à l'étude des centres destinés à l'observation des enfants irréguliers. Le Centre polyvalent de Lyon, Lyon, 1945.

FLOT Pierre, *Constatations médicales et sociales relatives à la délinquance juvénile en Bretagne, Paris, R. Foulon, 1945, 48 p.

FEXX Albert, Facteurs psychiques du terrain, Lyon, 1944.

FORRISSIER Paule, Foyer d'accueil avec centre d'observation et de triage pour les filles-mères et les pupilles de l'État. Utilité. Sa réalisation dans le département de l'Isère, Lyon, 1945.

GAMET André, *Contribution à l'étude de l'enfance coupable. Les facteurs familiaux et sociaux (A propos de la cartographie lyonnaise de la délinquance juvénile), Lyon, Emmanuel Vitte, 1941, 133 p.

GROSSE Georges, Protection médico-légale des enfants anormaux criminels, Paris, 1940.

HAUSER François, “Les réactions perverses chez les enfants, Paris, M. Vigné, 1939, $39 \mathrm{p}$.

JOUHON Henry, Contribution à l'étude des déséquilibres graves du caractère chez les adolescents. Les problèmes pratiques de leurs réactions antisociales, Paris, 1939.

LAVENANT René, Troubles de l'imagination. Mythomanie et bovarysme chez l'enfant et l'adolescent, Lyon, 1943.

MARREC Jean, *Homicide volontaire chez l'enfant. Contribution à l'étude de l'enfance coupable, Lyon, Bosc Frères \& L. Riou, 1943, 64 p.

MARTIN Pierre, "Contribution psychiatrique à l'étude de l'enfance coupable. Les mineures vagabondes et prostituées, Lyon, Noirclerc \& Fénétrier, 1939, $261 \mathrm{p}$.

MATHELIN Fernand, Contribution à l'étude de l'enfance délinquante. 
Centre médico-social de Dijon, Lyon, 1945.

MOLNAYER Georges, Examen et triage des mineurs délinquants. Essai de prophylaxie sociale appliquée au code pénal syrien, Lyon, Imprimerie des Facultés, 1940.

PESLE Georges-Dominique, "L'Enfance délinquante, vue d'un centre de triage, Paris, O.T.I., 1945, 67 p.

REY Guy, *Mineurs délinquants récidivistes. Contribution à l'étude de l'enfance coupable, Lyon, Bosc Frères \& L. Riou, 1943, 64 p.

VALET Raymond, *Contribution à l'étude du traitement et de l'assistance de l'enfance anormale. Le problème de l'adaptation sociale des enfants irréguliers, Lyon, Bosc Frères \& L. Riou, 1942, 68 p.

VANHEMS René, "Étude statistique portant sur 1.150 observations de mineurs délinquants réunis au Centre de triage de la Faculté de médecine de Lyon, Lyon, Imprimerie des Facultés, 1939, 138 p. 